\title{
Spirulina Prevents Memory Dysfunction, Reduces Oxidative Stress Damage and Augments Antioxidant Activity in Senescence- Accelerated Mice
}

\author{
Juen-Haur HWANG ${ }^{1,2}$, I-Te LeE ${ }^{3}$, Kee-Ching JENG ${ }^{4-6}$, Ming-Fu WANG ${ }^{7}$, Rolis Chien-Wei Hou ${ }^{8}$, \\ Su-Mei $\mathrm{WU}^{7}$ and Yin-Ching $\mathrm{CHAN}^{7, *}$ \\ ${ }^{1}$ Department of Otolaryngology, Buddhist Dalin Tzu-Chi General Hospital, Chiayi, Taiwan \\ ${ }^{2}$ School of Medicine, Tzu Chi University, Hualien, Taiwan \\ ${ }^{3}$ Division of Endocrinology and Metabolism, Taichung Veterans General Hospital, Taichung, Taiwan. \\ ${ }^{4}$ Applied Mathematics, Providence University, Shalu, Taiwan \\ ${ }^{5}$ Medical Technology, National Chung Hsing University, Taichung, Taiwan \\ ${ }^{6}$ Department of Education and Research, Taichung Veterans General Hospital, Taichung, Taiwan \\ ${ }^{7}$ Department of Food and Nutrition, Providence University, Shalu, Taichung, Taiwan \\ ${ }^{8}$ Department of Biotechnology, Yuanpei University, Hsinchu, Taiwan
}

(Received October 26, 2010)

\begin{abstract}
Summary Spirulina has proven to be effective in treating certain cancers, hyperlipidemia, immunodeficiency, and inflammatory processes. In this study, we aimed to investigate the effects of Spirulina on memory dysfunction, oxidative stress damage and antioxidant enzyme activity. Three-month-old male senescence-accelerated prone-8 (SAMP8) mice were randomly assigned to either a control group or to one of two experimental groups (one receiving daily dietary supplementation with $50 \mathrm{mg} / \mathrm{kg} \mathrm{BW}$ and one with $200 \mathrm{mg} / \mathrm{kg}$ BW of Spirulina platensis water extract). Senescence-accelerated-resistant (SAMR1) mice were used as the external control. Results showed that the Spirulina-treated groups had better passive and avoidance scores than the control group. The amyloid $\beta$-protein $(\mathrm{A} \beta)$ deposition was significantly reduced at the hippocampus and whole brain in both Spirulina groups. The levels of lipid peroxidation were significantly reduced at the hippocampus, striatum, and cortex in both Spirulina groups, while catalase activity was significantly higher only in the $200 \mathrm{mg} / \mathrm{kg}$ BW Spirulina group than in the control group. Glutathione peroxidase activity was significantly higher only in the cortex of the $200 \mathrm{mg} / \mathrm{kg}$ group than in that of the SAMP8 control group. However, superoxide dismutase activity in all parts of the brain did not significantly differ among all groups. In conclusion, Spirulina platensis may prevent the loss of memory possibly by lessening $\mathrm{A} \beta$ protein accumulation, reducing oxidative damage and mainly augmenting the catalase activity.
\end{abstract}

Key Words Spirulina platensis, memory dysfunction, amyloid $\beta$-protein, oxidative stress, antioxidant enzymes

Learning and memory dysfunctions are the main symptoms of Alzheimer's disease (AD), which is characterized by the deposition of amyloid $\beta$-protein $(\mathrm{A} \beta)$ in the brain. Alzheimer's patients also show an increased level of plasma thiobarbituric acid-reactive substances (TBARS), which indicates a higher oxidation of plasma unsaturated phospholipids, and an increased oxidation of red blood cell glutathione, which indicates oxidative stress in peripheral cells (1).

The senescence-accelerated prone mouse strain 8 (SAMP8) is often used as an animal model for $\operatorname{AD}(2,3)$. The SAMP8 has a shorter life span and shows many typical signs of aging, such as deficits in learning and memory, emotional disorders (increased anxiety-like behavior and depressive behavior) and altered circadian rhythm associated with certain pathological, biochemical and pharmacological changes, at an earlier age

E-mail: ycchan@pu.edu.tw than control mice (senescence-accelerated resistant mouse, SAMR1). Changes in gene expression of neuroprotection, signal transduction, protein folding/degradation, cytoskeleton/transport, immune response and reactive oxygen species (ROS) production have been reported in SAMP8 $(4,5)$. In addition, mitochondrial dysfunction was also noted in SAMP8 (6). Oxidative stress is believed to have an important role in AD development (4-7), and it is reasonable to expect that supplementation of antioxidants might prevent memory dysfunction.

Spirulina is a microscopic blue-green algae in the shape of a perfect spiral coil, living both in sea and fresh water. The most common Spirulina product for human and animal food supplements contains primarily two species: Spirulina platensis and Spirulina maxima. It is a rich and valuable food source of macro- and micronutrients including high quality protein, iron, gammalinolenic fatty acid, carotenoids, vitamins $\mathrm{B}_{1}$ and $\mathrm{B}_{2}$, 
minerals, C-phycocyanin (CPC), etc. (8). It has been reported that Spirulina platensis or its active ingredient (CPC) exerts anti-oxidative activity, anti-lipid peroxidation, anti-inflammatory effects, and inhibitory effects on prostaglandin and leukotriene biosynthesis $(9,10)$.

In vivo and in vitro studies have proven the effectiveness of Spirulina for certain cancers, hepatotoxicity, hyperglycemia, hyperlipidemia, immunodeficiency, and inflammatory processes (11). However, the effects of Spirulina on memory dysfunction and antioxidant enzyme activity have not been reported. As we know, the hippocampus, striatum, and cortex are key brain structures implicated in cognition and/or memory functions (12-16). Thus, in this study, we aimed to investigate these issues in SAMP8 mice.

\section{MATERIALS AND METHODS}

Animals and food intake. SMAP8 and SAMR1 mice were procured from Kyoto University, Japan, and were maintained by inbred breeding in the standard animal room of Providence University. Male SAMP8 mice ( 3 mo old) were randomly divided into three groups ( $n=8$ each): one control and two Spirulina (50 and $200 \mathrm{mg} / \mathrm{kg}$ BW per day) groups. Male SAMR1 mice, which showed normal characteristics, were used as the external control. The mice were housed about 5 per cage under controlled environmental conditions $\left(22 \pm 2^{\circ} \mathrm{C}, \quad 65 \pm 5 \%\right.$ relative humidity, $0700-1900 \mathrm{~h}$ lighting period). Animals were allowed free access to drinking water and the AIN 93-M basal diet for $12 \mathrm{wk}$.

For the two Spirulina groups, we used the water extracts of Spirulina platensis supplied by Far East BioTec Co., Ltd. as experimental materials. The water extract of Spirulina platensis contained 15 $\pm 5 \%$ CPC and $35 \pm 5 \%$ polysaccharide after dialysis to remove molecules less than $3 \mathrm{kDa}$ and lyophilization. As in previous studies $(11,17)$, the final concentration of Spirulina platensis extract was administered orally with food at a concentration of 50 or $200 \mathrm{mg} / \mathrm{kg}$ BW per day. To assure the potency of Spirulina, we checked the concentration of phycocyanin, a biliprotein pigment found in Spirulina platensis, in the whole diet $1 \mathrm{~d}$ after exposure to light. The results revealed the water extract of Spirulina platensis remained stable under controlled environmental conditions $\left(22 \pm 2^{\circ} \mathrm{C}, \quad 65 \pm 5 \%\right.$ relative humidity, 0700-1900 h lighting period). Therefore, the diet containing Spirulina water extract at the prescribed concentrations was replaced every other day. The food intake of the mice was recorded every day, and the mice were weighed weekly. The study protocol was approved by the animal research ethics committee at Providence University, Taichung, Taiwan.

Memory evaluation. One week before the memory function evaluation, the mice were individually placed in cubic boxes (each side, $25 \mathrm{~cm}$ ). Ambulatory activity of all mice was measured for $10 \mathrm{~min}$ using a video activity monitor (model E61-21, Coulbourn Instruments, Philadelphia, PA). Mice with poor activity were excluded from subsequent tests.

Behavioral studies were performed in a shuttle box
$(35 \times 17 \times 20 \mathrm{~cm}$, model E10-15, Coulbourn Instruments) that consisted of two equal compartments connected by a small opening $(7.5 \times 6.5 \mathrm{~cm}$, Guillotine door, model E10-15GD, Coulbourn Instruments). One compartment was lit, while the other was darkened by a black semi-transparent plastic cover. The floor of the box was a platform of steel rods.

The single-trial passive avoidance test used a shuttle box $(35 \times 17 \times 20 \mathrm{~cm}$ : width $\times$ length $\times$ height, model E10-15, Coulbourn Instruments) that consisted of two equal compartments connected by a small opening $(7.5 \times 6.5 \mathrm{~cm}$, Guillotine door, model E10-15, GD, Coulbourn Instruments). One of the compartments was lighted while the other compartment was darkened by covering the top with a black semi-transparent plastic. The floor of the box was a platform of steel rods. In the acquisition trial, each mouse exposed to this test was placed in the lighted compartment, and after a brief orientation period $(10 \mathrm{~s})$, the partition of the opening was raised to allow the mouse to explore the apparatus freely. The mice would suffer an electric foot shock ( $3 \mathrm{~mA})$ for $3 \mathrm{~s}$ through the stainless steel grid floor when it entered the dark compartment. After $24 \mathrm{~h}$, the retention trial was performed in the same manner; the mice were put into the bright compartment again, and the time when they entered the dark compartment was recorded. The time of the retention trial was $180 \mathrm{~s}$.

Active (shuttle) avoidance test examined the successful avoidance response which was conducted if a tested mouse moved itself from one compartment to another compartment within the shuttle box after receiving a conditional stimulus (CS), $10 \mathrm{~s}$ of tone and red, yellow and green light. If the tested mouse did not perform a successful avoidance response, an unconditional stimulus (UCS), a $0.3 \mathrm{~mA}, 5 \mathrm{~s}$ scrambled foot shock, would be given during the CS presentation. The test was conducted for $2 \mathrm{~d}$ including one acquisition trial and one testing trial. Each mouse received four daily sessions of a combination of $5 \mathrm{CS} / \mathrm{UCS}$ trials, a total of 20 trials. Between the sessions the tested mouse was allowed to rest for 15-20 $\mathrm{min}$. The avoidance responses of tested mice were recorded automatically (18).

Measurements of brain $A \beta$ deposition. After the memory evaluation, the mice were sacrificed. Each brain was quickly dissected from the skull and individually fixed in a $10 \%$ buffered neutral formalin solution for $1 \mathrm{wk}$. The brain was sectioned ( $5-\mu \mathrm{m}$ thickness) using a microtome (RM 2145, Leica, Nussloch, Germany) based on the method described by Popesko et al. (19).

$\beta$-Amyloid in the B-section of brains was stained by the immunohistochemical method of Popesko et al. (19) using an UltraTech H (DAB) Streptavidin-Biotin Universal Detection System (Immunotech, Cedex, France). To identify $\beta$-amyloid plaque, sections were immersed in $10 \mathrm{mmol}$ citrate buffer and microwaved three times (each for $2 \mathrm{~min}$ ), incubated with primary anti- $\beta$ amyloid antibody (1:300; MAb to $\beta$-amyloid a.a. $17-24$, Biodesign International, Kennebunk, ME, USA) for $2 \mathrm{~h}$ at room temperature, incubated in polyvalent-biotinylated antibody (goat anti-rabbit antibodies, $1: 1,000$ ) 
for $45 \mathrm{~min}$, incubated in DAB substrate solution for $30 \mathrm{~min}$, and stained with Mayer's hematoxylin (Sigma, St. Louis, MO, USA) for $3 \mathrm{~min}$. Areas of $\beta$-amyloid appeared brownish in color.

The $A \beta$ positive areas in sections of the hippocampus and whole brain were measured according to Lim et al. (20) with slight modification. Briefly, $\mathrm{A} \beta$ positive areas were measured in sections from the middle part of the brain under $100 \times$ magnification, and more than 20 fields were assessed using an image analyzer (Q500MC, Leica).

Redox status analysis. After sacrificing the mice, the hippocampus, striatum, and cortex were separated, immediately placed in sodium phosphate buffer (100 $\mathrm{mM} / \mathrm{L} ; \mathrm{pH}$ 7.4), homogenized, and centrifuged at $3,000 \times g$ for $10 \mathrm{~min}$ in a refrigerated centrifuge (Hettich Universal 16 R, Tuttlingen, Germany). The supernatants were collected and assayed for lipid peroxide, superoxide dismutase (SOD), catalase (CAT), and glutathione peroxidase (GSH-Px) activity.

The level of lipid peroxidation in each tissue was determined according to the method described by Ohkawa et al. (21). The supernatants were mixed with $2^{\prime}$-thiobarbituric acid $(4 \mathrm{~g} / \mathrm{kg}$ in $0.2 \mathrm{M} \mathrm{HCl})$ and butylated hydroxytoluene ( $2 \mathrm{~g} / \mathrm{kg}$ in $95 \%$ ethanol) at a ratio of $1: 2: 0.3$, and then the mixture was heated at $90^{\circ} \mathrm{C}$ for $45 \mathrm{~min}$, cooled down, and mixed with $5 \mathrm{~mL}$ of $n$ butanol. The $n$-butanol layer was separated by centrifugation $(1,000 \times g$ for $10 \mathrm{~min})$ and assayed for TBARS spectrophotometrically at $532 \mathrm{~nm}$. The results were expressed as $\mu \mathrm{mol}$ equivalents of malondialdehyde per $\mathrm{g}$ of tissue, using malondialdehyde from tetramethoxypropane as the standard and double-distilled water as the control.

CAT activity was measured using the method of Aebi (22). Changes in absorbance of a mixture consisting of $1.0 \mathrm{~mL}$ of $\mathrm{H}_{2} \mathrm{O}_{2}(30 \mathrm{mM})$ and $2 \mathrm{~mL}$ of supernatant were recorded at $240 \mathrm{~nm}$ in a spectrophotometer at $25^{\circ} \mathrm{C}$. CAT activity was calculated in terms of moles of $\mathrm{H}_{2} \mathrm{O}_{2}$ consumed $/ \mathrm{min} / \mathrm{g}$ of tissue protein.

GSH-Px activity was measured using the method of Paglia and Valentine (23). Briefly, the production of formazan in a mixture consisting of $200 \mu \mathrm{L}$ of glutathione reductase ( $5 \mathrm{U} / \mathrm{mL}$; GSSG-R), $50 \mu \mathrm{L}$ of glutathione (40 mM; GSH), $620 \mu \mathrm{L}$ of phosphate buffer $(0.25 \mathrm{M}$; $\mathrm{pH}$ 7.4), $100 \mu \mathrm{L}$ of superantant, $10 \mu \mathrm{L}$ of $\beta$-nicotinamide adenine dinucleotide phosphate reduced tetrasodium salt ( $20 \mathrm{mM}$; NADPH), and $20 \mu \mathrm{L}$ of cumene hydroperoxide $(15 \mathrm{mM})$ was determined at $340 \mathrm{~nm}$ in a spectrophotometer at $25^{\circ} \mathrm{C}$. One unit of GSH-Px was defined as the number of $\mu \mathrm{mol}$ of NADPH oxidized per minute at $25^{\circ} \mathrm{C}$.

The SOD activity was measured using the method of Marklund and Marklund (24). The production of formazan in a mixture containing $10 \mu \mathrm{L}$ of supernatant, $50 \mathrm{~mm}$ Tris-HCl $(\mathrm{pH} 8.2)$, and $50 \mathrm{~mm}$ pyrogallol in a final volume of $3.017 \mathrm{~mL}$ was determined at $325 \mathrm{~nm}$ in a spectrophotometer at $25^{\circ} \mathrm{C}$. One unit of SOD was defined as the amount of protein that inhibits the rate of pyrogallol auto-oxidation by $50 \%$. Total SOD activity

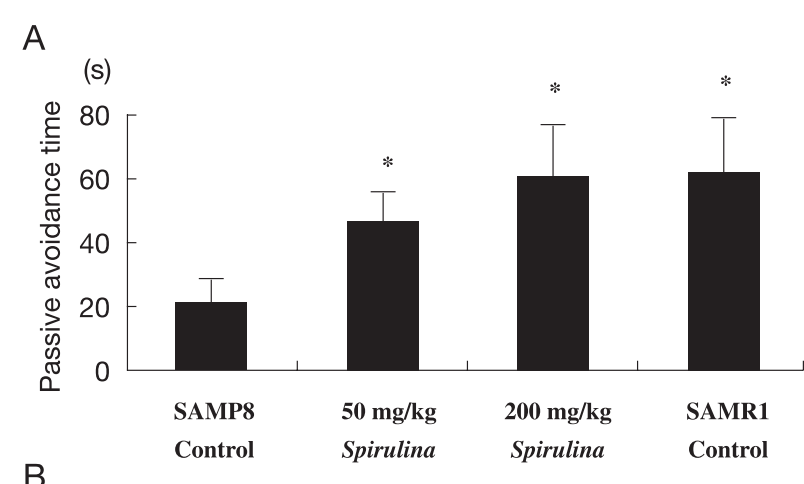

B

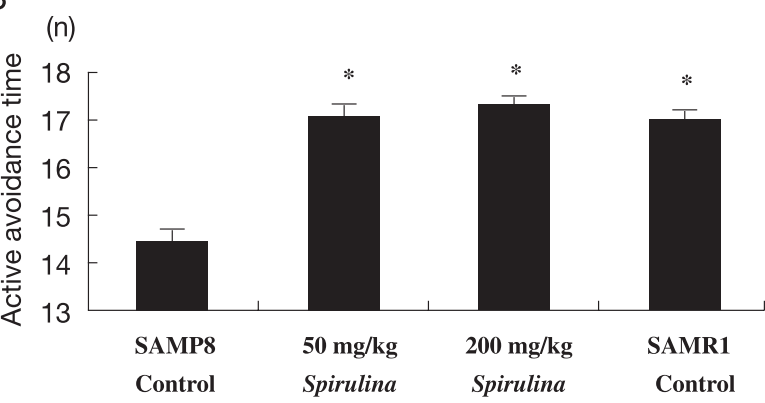

Fig. 1. Memory evaluation in 6-mo-old male SAMP8 mice fed different diets. The passive avoidance time (A) and active avoidance time (B) were longer or higher in both Spirulina groups than in the SAMP8 control group. Both avoidances in the $200 \mathrm{mg} / \mathrm{kg}$ group were similar to that in the SAMR1 group. Each value is the mean \pm SE of $n=8$ /group. *Indicates significant difference from the SAMP8 control group.

but not the activities of SOD isoforms were measured.

CAT, GSH-Px, and SOD activities are expressed as units/g protein. Soluble protein concentration in the samples was determined using the Pierce Micro BCA protein assay kit.

Statistical analysis. All data are expressed as mean \pm standard error of mean and analyzed using the SPSS 10.0 software package (SPSS Inc., Chicago, IL, USA). Data were evaluated by independent samples $t$-test and one-way ANOVA. $p<0.05$ was considered statistically significant.

\section{RESULTS}

Spirulina does not affect body weight, food intake, or ambulatory activity

Body weight, food intake, and ambulatory activity were not significantly different among the three groups (one-way ANOVA, $p>0.05$ ) (data not shown).

Spirulina improved memory function

Compared to the SAMP8 control group, both Spirulina groups had significantly prolonged passive avoidance time (independent samples $t$-test, $p<0.05$ for both groups; Fig. 1A) and active avoidance time (independent samples $t$-test, $p<0.05$, for both groups; Fig. 1B) on the next day. Moreover, the passive or active avoidance time of both Spirulina groups was similar to that of the SAMR1 control group (independent samples $t$-test, $p>0.05$ for both groups in each test). These results indicated that the addition of Spirulina platensis to the diet could improve the emotional memory of SAMP8 mice. 
A

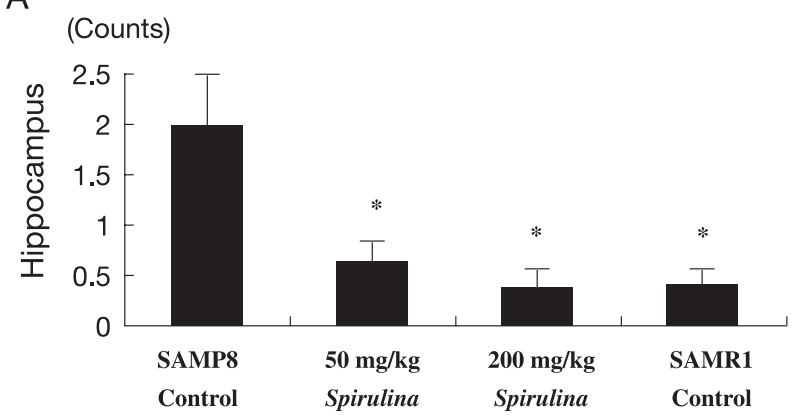

B

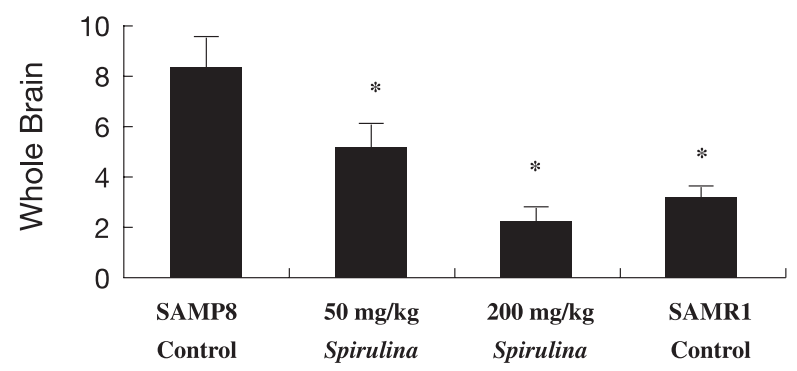

Fig. 2. $\beta$-Amyloid $(\mathrm{A} \beta)$ deposition in the brains of 6 mo-old SAMP8 mice fed different diets. $\mathrm{A} \beta$ accumulation in the hippocampus (A) and whole brain (B) is lower in the $50 \mathrm{mg} / \mathrm{kg} \mathrm{BW}$ Spirulina group, $200 \mathrm{mg} / \mathrm{kg}$ BW Spirulina group, and the SAMR1 group than in the SAMP8 control group. Each count is the mean \pm SE of $n=8$ /group. A $\beta$ accumulation was assessed under $100 \times$ magnification by photomicroscopy. ${ }^{*}$ Indicates significant difference from the SAMP8 control group.

Spirulina reduced the accumulation of $A \beta$ deposition in the hippocampus and whole brain

$\mathrm{A} \beta$ accumulation in the hippocampus (Fig. 2A) and whole brain (Fig. 2B) was markedly lower in both Spirulina groups than in the SAMP8 control group (independent samples $t$-test, $p<0.05$, for both groups), indicating that the Spirulina supplements can reduce accumulation of $A \beta$ in the hippocampus and whole brain.

Spirulina enhanced the redox status

Compared to the SAMP8 control group, both Spirulina groups had significantly lower levels of lipid peroxides in the hippocampus $(0.67 \pm 0.10,0.64 \pm 0.06$ and $1.13 \pm 0.05$, respectively, for the $50 \mathrm{mg} / \mathrm{kg}, 200 \mathrm{mg} / \mathrm{kg}$, and SAMP8 control groups), striatum (0.91 \pm 0.09 , $0.72 \pm 0.054$, and $1.15 \pm 0.053$, respectively, for the $50 \mathrm{mg} / \mathrm{kg}, 200 \mathrm{mg} / \mathrm{kg}$, and SAMP8 control groups), and cortex $(0.67 \pm 0.050,0.66 \pm 0.053$, and $1.13 \pm$ 0.15 , respectively, for the $50 \mathrm{mg} / \mathrm{kg}, 200 \mathrm{mg} / \mathrm{kg}$, and SAMP8 control groups; all means compared by the independent samples $t$-test, $p<0.05$ for both groups; Fig. 3) and had significantly higher CAT activity in the hippocampus $(3.16 \pm 0.38$ for the $200 \mathrm{mg} / \mathrm{kg}$ group versus $2.21 \pm 0.088$ for the SAMP8 control group), striatum $(2.95 \pm 0.30$ versus $1.89 \pm 0.14)$, and cortex

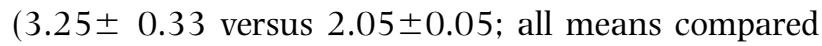
by the independent samples $t$-test, $p<0.05$ for both groups; Fig. 4). GSH-Px activity was significantly higher

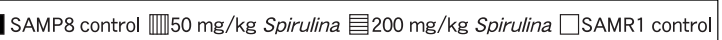

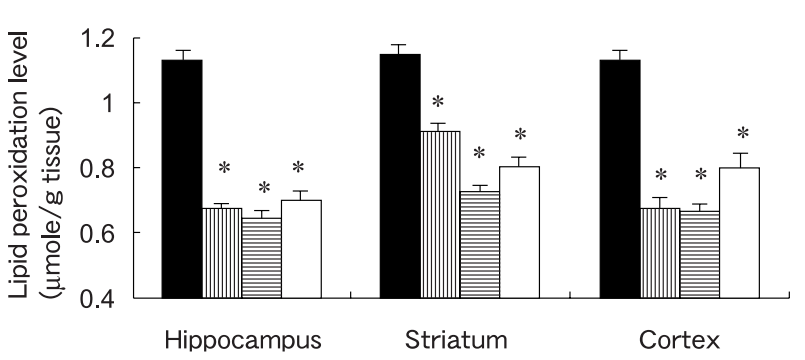

Fig. 3. The level of lipid peroxidation in 6-mo-old male SAMP8 mice fed different diets. Lipid peroxide level was reduced in the brain hippocampus, striatum, and cortex of mice in the $50,200 \mathrm{mg} / \mathrm{kg}$ BW Spirulina groups and SAMR1 control group (when compared to that of the SAMP8 control group). Each value is the mean \pm SE of $n=8$ /group. *Indicates significant difference from the SAMP8 control group.

SAMP8 control $\mathbb{\| m} 50 \mathrm{mg} / \mathrm{kg}$ Spirulina 邑 $200 \mathrm{mg} / \mathrm{kg}$ Spirulina $\square$ SAMR1 control

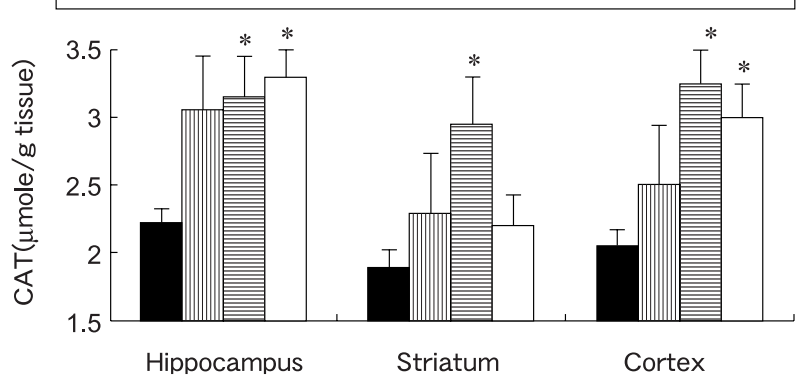

Fig. 4. CAT activity in 6-mo-old male SAMP8 mice fed different diets. Activity was higher in the brain hippocampus, striatum, and cortex of mice in the $200 \mathrm{mg} / \mathrm{kg}$ BW Spirulina group and SAMR1 control group, but not the $50 \mathrm{mg} / \mathrm{kg}$ BW Spirulina group (when compared to that of the SAMP8 control group). Each value is the mean \pm SE of $n=8$ /group. *Indicates significant difference from the SAMP8 control group.

only in the cortex of the $200 \mathrm{mg} / \mathrm{kg}$ group than in that of the SAMP8 control group (18.12 \pm 2.17 versus $10.00 \pm 2.63$; independent samples $t$-test, $p<0.05)$. However, SOD activity in all parts of the brain did not significantly differ between the $200 \mathrm{mg} / \mathrm{kg}$ group and SAMP8 control group (data not shown; independent samples t-test, $p<0.05)$. In addition, anti-oxidant enzyme activities of the $50 \mathrm{mg} / \mathrm{kg}$ group were similar to those of the SAMP8 control group in all parts of the brain (data not shown; independent samples t-test, $p>0.05)$. These results indicated that high-dose Spirulina supplements could reduce oxidative stress damage and augment CAT activity mainly.

\section{DISCUSSION}

This experimental study showed that dietary supplementation with $200 \mathrm{mg} / \mathrm{kg}$ BW Spirulina platensis water extracts significantly slowed loss of memory by lessening $A \beta$ protein accumulation, reducing oxidative damage, and augmenting the catalase activity in SAMP8, 
an animal model for AD.

Experimentally and clinically it is well known that a primary source of ROS generation is through oxidative phosphorylation, ischemia/reperfusion or prolonged hypoperfusion, such as that seen in myocardial infarction, cerebrovascular accidents, aging, and presbyacusis. Although the exact mechanisms of $\mathrm{AD}$ are still unclear, it has been reported that $\mathrm{A} \beta$ and $\mathrm{A} \beta$-related reactive oxidative species play a critical role in the development of $\mathrm{AD}$ (25). In addition, reduced activities of endogenous enzymes, such as superoxide dismutase (SOD), catalase and glutathione peroxidase, and/or low intake of a variety of small molecules with anti-oxidative activity in the human diet, might lead to the development of AD. Liu and Mori (7) showed that cognitive dysfunction in senescence-accelerated mice correlates well with elevation in lipid peroxidation level and decrease in glutathione (GSH) level in the brain.

On the other hand, Massaad et al. (15) demonstrated that over-expression of SOD could prevent AD-related learning and memory deficits by decreasing hippocampal superoxide, and reducing $A \beta$ plaques in the $\operatorname{Tg} 2576$ mouse. In this study, Spirulina water extracts reduced $\mathrm{A} \beta$ accumulation in the hippocampus and whole brain, and decreased lipid peroxidation in the hippocampus, striatum, and cortex. Besides, Spirulina had differential effects on the activity of antioxidant enzymes. Notably, the high dose of Spirulina platensis extract enhanced CAT activity in the hippocampus, striatum, and cortex, and enhanced GSH-Px activity in the cortex, but had no affect on SOD activity anywhere in the brain.

In addition to antioxidant capability, the detailed neuroprotective mechanisms of Spirulina water extracts were not very clear. Wang et al. (26) reported that Spirulina can reduce the ischemia/reperfusion-induced apoptosis and cerebral infarction in mice with focal ischemia. Spirulina can also enhance striatal dopamine recovery and induce rapid, transient microglia activation after injury of the rat nigrostriatal dopamine system (27). CPC attenuated doxorubicin-induced reactive oxygen species formation, Bax protein activity, mitochondrial cytochrome $c$ release, caspase-3 activity, DNA fragmentation, and apoptosis via suppression of p38 MAPK and NF- $\kappa$ B signal pathway in cardiomyocytes (28). CPC also reduced apoptosis of R-HepG2 cells through up-regulation of the Bcl-2 protein and downregulation of the Bax protein (29). However, more study will be needed to link CPC-induced improvement in emotional memory function to changes in signaling pathways.

\section{CONCLUSIONS}

Dietary supplementation with Spirulina plantensis ameliorates deterioration of memory in SAMP8 mice via reducing $\mathrm{A} \beta$ accumulation, lowering the level of lipid peroxidation, and augmenting the antioxidant system, especially CAT activity, all of which might have an important role in the prevention of $\mathrm{AD}$.

\section{Competing interests}

The authors declare that they have no competing interest.

\section{Author's contribution}

YCC conceived of and designed the study. SMW performed most of the experiments. JHH and KCJ analyzed the experimental results. JHH and YCC drafted the manuscript. ITL, MFW, RCWH, and YCC supervised the study. All authors participated in the critical revision of the manuscript; and all authors have given final approval of the version to be published.

\section{Acknowledgments}

We are grateful to Far East Bio-Tec Co., Ltd., Taiwan, for providing the water extract of Spirulina platensis. This study was supported by grants from the National Science Council, Taiwan (NSC-95-231693-B-126-008) and the Taichung Veterans General Hospital and Providence University (TCVGH-PU988104), Taichung, Taiwan.

\section{REFERENCES}

1) Viña J, Lloret A, Ortí R, Alonso D. 2004. Molecular bases of the treatment of Alzheimer's disease with antioxidants: prevention of oxidative stress. Mol Aspects Med 25: 117-123.

2) Takeda T, Hosokawa M, Takeshita S, Irino M. 1981. A new murine model of accelerated senescence. Mech Ageing Dev 17: 183-194.

3) Takeda T. 2009. Senescence-accelerated mouse (SAM) with special references to neurodegeneration models, SAMP8 and SAMP10 mice. Neurochem Res 34: 639659.

4) Butterfield DA, Poon HF. 2005. The senescence-accelerated prone mouse (SAMP8): a model of age-related cognitive decline with relevance to alterations of the gene expression and protein abnormalities in Alzheimer's disease. Exp Gerontol 40: 774-783.

5) Tha KK, Okuma Y, Miyazaki H, Murayama T, Uehara T, Hatakeyama R, Hayashi Y, Nomura Y. 2000. Changes in expressions of proinflammatory cytokines IL-1beta, TNF-alpha and IL-6 in the brain of senescence accelerated mouse (SAM) P8. Brain Res 885: 25-31.

6) Nakahara H, Kanno T, Inai Y, Utsumi K, Hiramatsu M, Mori A, Packer L. 1998. Mitochondrial dysfunction in the senescence accelerated mouse (SAM). Free Radic Biol Med 24: 85-92.

7) Liu J, Mori A. 1993. Age-associated changes in superoxide dismutase activity, thiobarbituric acid reactivity and reduced glutathione level in the brain and liver in senescence accelerated mice (SAM): a comparison with ddY mice. Mech Ageing Dev 71: 23-30.

8) Chamorro G, Salazar M, Araújo KG, dos Santos CP, Ceballos G, Castillo LF. 2002. Update on the pharmacology of Spirulina (Arthrospira), an unconventional food. Arch Latinoam Nutr 52: 232-240.

9) Bhat VB, Madyastha KM. 2000. C-phycocyanin: a potent peroxyl radical scavenger in vivo and in vitro. Biochem Biophys Res Commun 275: 20-25.

10) Chaiklahan R, Chirasuwan N, Siangdung W, Paithoonrangsarid K, Bunnag B. 2010. Cultivation of Spirulina platensis using pig wastewater in a semi-continuous 
process. J Microbiol Biotechnol 20: 609-614.

11) Chamorro G, Perez-Albiter M, Serrano-Garcia N, MaresSamano JJ, Rojas P. 2006. Spirulina maxima pretreatment partially protects against 1-methyl-4-phenyl1,2,3,6-tetrahydropyridine neurotoxicity. Nutr Neurosci 9: 207-212.

12) Dalgleish T. 2004. The emotional brain. Nat Rev Neurosci 5: 583-589.

13) Wittmann BC, Schiltz K, Boehler CN, Düzel E. 2008. Mesolimbic interaction of emotional valence and reward improves memory formation. Neuropsychologia 46: 1000-1008.

14) Ferreira TL, Shammah-Lagnado SJ, Bueno OF, Moreira KM, Fornari RV, Oliveira MG. 2008. The indirect amygdala-dorsal striatum pathway mediates conditioned freezing: insights on emotional memory networks. Neuroscience 153: 84-94.

15) Massaad CA, Washington TM, Pautler RG, Klann E. 2009. Overexpression of SOD-2 reduces hippocampal superoxide and prevents memory deficits in a mouse model of Alzheimer's disease. Proc Natl Acad Sci USA 106: $13576-13581$.

16) Gu Y, Huang CS, Inoue T, Yamashita T, Ishida T, Kang KM, Nakao A. 2010. Drinking hydrogen water ameliorated cognitive impairment in senescence-accelerated mice. J Clin Biochem Nutr 46: 269-276.

17) Thaakur SR, Jyothi B. 2007. Effect of Spirulina maxima on the haloperidol induced tardive dyskinesia and oxidative stress in rats. J Neural Transm 114: 1217-1225.

18) Flood JF, Morley JE. 1993. Age-related changes in footshock avoidance acquisition and retention in senescence-accelerated mouse (SAM). Neurobiol Aging 14: 153-157.

19) Popesko P, Rajtová V, Horák J. 1992. A Colour Atlas of the Anatomy of Small Laboratory Animals, p 105-166. Wolfe Publishing, London.

20) Lim GP, Yang F, Chu T, Chen P, Beech W, Teter B, Tran T, Ubeda O, Hsiao Ashe K, Frautschy SA, Cole GM. 2000. Ibuprofen suppresses plaque pathology and inflammation in a mouse model for Alzheimer's Disease. J Neuro- sci 20: 5709-5714.

21) Ohkawa H, Ohishi N, Yagi K. 1979. Assay for lipid peroxides in animal tissues by thiobarbituric acid reaction. Anal Biochem 95: 351-358.

22) Aebi H. 1983. Methods of enzymatic analysis. In: Catalase (Bergmeyer H, ed), p 673-686. Verlag Chemie Weinhein, Deerfield Beach, FL.

23) Paglia DE, Valentine WN. 1967. Studies on the qualitative characterization of erythrocyte glutathione peroxidase. J Lab Clin Med 70: 159-169.

24) Marklund S, Marklund G. 1974. Involvement of the superoxide anion radical in the autoxidation of pyrogallol and a convenient assay for superoxide dismutase. Eur J Biochem 47: 469-474.

25) Pappolla MA, Chyan YJ, Omar RA, Hsiao K, Perry G, Smith MA, Bozner P. 1998. Evidence of oxidative stress and in vivo neurotoxicity of $\beta$-amyloid in a transgenic mouse model of Alzheimer's disease. Am J Pathol 152: 871-877.

26) Wang Y, Chang CF, Chou J, Chen HL, Deng X, Harvey BK, Cadet JL, Bickford PC. 2005. Dietary supplementation with blueberries, spinach, or Spirulina reduces ischemic brain damage. Exp Neurol 193: 75-84.

27) Strömberg I, Gemma C, Vila J, Bickford PC. 2005. Blueberry- and Spirulina-enriched diets enhance striatal dopamine recovery and induce a rapid, transient microglia activation after injury of the rat nigrostriatal dopamine system. Exp Neurol 196: 298-307.

28) Khan M, Varadharaj S, Ganesan LP, Shobha JC, Naidu MU, Parinandi NL, Tridandapani S, Kutala VK, Kuppusamy P. 2006. C-phycocyanin protects against ischemia-reperfusion injury of heart through involvement of p38 MAPK and ERK signaling. Am J Physiol Heart Circ Physiol 290: 2136-2145.

29) Roy KR, Arunasree KM, Dhoot A, Aparna R, Reddy GV, Vali S, Reddanna P. 2007. C-Phycocyanin inhibits 2acetylaminofluorene-induced expression of MDR1 in mouse macrophage cells: ROS mediated pathway determined via combination of experimental and in silico analysis. Arch Biochem Biophys 459: 169-177. 